\title{
Video Article \\ Laser Ablation of the Zebrafish Pronephros to Study Renal Epithelial Regeneration
}

\author{
Corbin S. Johnson ${ }^{* 1}$, Nicholas F. Holzemer* ${ }^{1}$, Rebecca A. Wingert ${ }^{1}$ \\ ${ }^{1}$ Department of Biological Sciences, University of Notre Dame \\ *These authors contributed equally
}

Correspondence to: Rebecca A. Wingert at rwingert@nd.edu

URL: https://www.jove.com/video/2845

DOI: doi:10.3791/2845

Keywords: Developmental Biology, Issue 54, kidney, zebrafish, regeneration, epithelium, acute kidney injury, ablation

Date Published: 8/29/2011

Citation: Johnson, C.S., Holzemer, N.F., Wingert, R.A. Laser Ablation of the Zebrafish Pronephros to Study Renal Epithelial Regeneration. J. Vis. Exp. (54), e2845, doi:10.3791/2845 (2011).

\section{Abstract}

Acute kidney injury (AKI) is characterized by high mortality rates from deterioration of renal function over a period of hours or days that culminates in renal failure ${ }^{1}$. AKI can be caused by a number of factors including ischemia, drug-based toxicity, or obstructive injury ${ }^{1}$. This results in an inability to maintain fluid and electrolyte homeostasis. While AKI has been observed for decades, effective clinical therapies have yet to be developed. Intriguingly, some patients with AKI recover renal functions over time, a mysterious phenomenon that has been only rudimentally characterized $^{1,2}$. Research using mammalian models of AKI has shown that ischemic or nephrotoxin-injured kidneys experience epithelial cell death in nephron tubules ${ }^{1,2}$, the functional units of the kidney that are made up of a series of specialized regions (segments) of epithelial cell types $^{3}$. Within nephrons, epithelial cell death is highest in proximal tubule cells. There is evidence that suggests cell destruction is followed by dedifferentiation, proliferation, and migration of surrounding epithelial cells, which can regenerate the nephron entirely ${ }^{1,2}$. However, there are many unanswered questions about the mechanisms of renal epithelial regeneration, ranging from the signals that modulate these events to reasons for the wide variation of abilities among humans to regenerate injured kidneys.

The larval zebrafish provides an excellent model to study kidney epithelial regeneration as its pronephric kidney is comprised of nephrons that are conserved with higher vertebrates including mammals ${ }^{4,5}$. The nephrons of zebrafish larvae can be visualized with fluorescence techniques because of the relative transparency of the young zebrafish ${ }^{6}$. This provides a unique opportunity to image cell and molecular changes in realtime, in contrast to mammalian models where nephrons are inaccessible because the kidneys are structurally complex systems internalized within the animal. Recent studies have employed the aminoglycoside gentamicin as a toxic causative agent for study of AKI and subsequent renal failure: gentamicin and other antibiotics have been shown to cause AKI in humans, and researchers have formulated methods to use this agent to trigger kidney damage in zebrafish ${ }^{7,8}$. However, the effects of aminoglycoside toxicity in zebrafish larvae are catastrophic and lethal, which presents a difficulty when studying epithelial regeneration and function over time. Our method presents the use of targeted cell ablation as a novel tool for the study of epithelial injury in zebrafish. Laser ablation gives researchers the ability to induce cell death in a limited population of cells. Varying areas of cells can be targeted based on morphological location, function, or even expression of a particular cellular phenotype. Thus, laser ablation will increase the specificity of what researchers can study, and can be a powerful new approach to shed light on the mechanisms of renal epithelial regeneration. This protocol can be broadly applied to target cell populations in other organs in the zebrafish embryo to study injury and regeneration in any number of contexts of interest.

\section{Video Link}

The video component of this article can be found at https://www.jove.com/video/2845/

Protocol

\section{Microinjection procedure to label the zebrafish pronephros proximal tubule epithelium}

For this procedure, we use a microinjection system (Harvard Appartus, PL190), with pressurized air supplied by an air compressor (Jun-Air, Model 6-4), and micromanipulator apparatus (Narishege parts MN151, IP3, and GJI) that are assembled at a stereomicroscope station (Nikon, SMZ-Zoom 645) as per manufacturer instructions. Dextran conjugates are readily endocytosed by the nephron proximal tubule, enabling preferential labeling of this epithelial cell population ${ }^{9}$.

1. Exposure to methylene blue can accentuate the autofluorescence of the yolk sac and makes visualization of the nephron tubules more difficult.

2. Raise the fertilized embryos at $28.5^{\circ} \mathrm{C}$ to a developmental timepoint between $48-55$ hours post fertilization (hpf). This is the ideal stage to perform larvae microinjections because of the ease at which intramuscular microinjection can be performed, and because renal function commences at this time. 
3. Remove the chorion from any unhatched zebrafish using a pair of fine forceps. Rinse the chorion debris from the petri dish and refill the dish with $30 \mathrm{mLs}$ of modified E3 solution.

4. Prepare an injection tray for the embryos. Our preferred injection mold is made of $1.5 \%$ agarose/E3, in which triangular depressions are formed such that the embryo can be positioned inside for injection. To make the injection mold, we float a plastic mold with wedge shaped protrusions (described in detail previously ${ }^{11}$ ) in a Petri dish filled with a base of $1.5 \%$ agarose.

5. Prepare glass microinjection needles by pulling capillary tubes with a needle puller, as described in the Zebrafish Book ${ }^{10}$. In brief, pull the glass capillary tubes then use fine metal forceps to cut the tip of the microinjection needle to make a tip with a sharp point while viewing the needle tip under a stereomicroscope. Store the cut needles covered in a petri dish and positioned on modeling clay to protect the cut edge and prevent dust accumulation.

6. Prepare the injection solution to label the proximal tubule. Dissolve $40 \mathrm{kD}$ dextran-fluorescein conjugate (Invitrogen, D1845) at a concentration of $1 \mathrm{mg} / \mathrm{mL}$ in distilled water.

7. To anesthetize the fish, add $5 \mathrm{mLs}$ of $0.2 \%$ Tricaine $\mathrm{pH} 7.0$ to the petri dish containing the zebrafish larvae in $30 \mathrm{mLs}$ of E3. The fish are anesthetized when they no longer exhibit the touch response. It is vital that the fish are completely anesthetized or they will twitch upon attempting to inject them.

8. Transfer the anesthetized fish to an injection mold tray using a plastic transfer pipet. Position the animal with its head in the deepest part of the triangular depression well, and on its side such that the trunk is resting along the angled side of the well.

9. Perform intramuscular microinjection. Load a cut needle with 2-3 $\mu \mathrm{L}$ of dextran fluorescein, and inject the animal in a trunk somite with approximately $1 \mathrm{~nL}$ of solution. Aim for the upper portion of the somite, and avoid the yolk sac extension. This ensures that the nephron tubules are not mechanically disrupted by the microinjection process.

10. Transfer injected zebrafish to a clean petri dish, and add modified E3 solution. Rinse the animals 3 times with fresh E3 to remove all traces of Tricaine. Cover the lid of the petri dish with aluminum foil to provide light-protection for the dextran, and return the animals to the $28^{\circ} \mathrm{C}$ incubator overnight.

\section{Targeted laser ablation of proximal tubule cells}

For this procedure, we use a stereomicroscope with epifluorescence to score animals with dextran fluorescein brightly-labeled proximal tubules and select these for laser ablation. Then, we use a pulsed micropoint laser system (Photonic Instruments, Inc) that has been attached to a compound microscope (Nikon Eclipse 80i with epifluorescent attachment), and calibrated for use as per manufacturer instructions, to perform nephron cell ablation. We have had the most success in cell ablation by using a FITC filter that enables the researcher to view the fluorescent tubule regions under brightfield lighting.

In advance of this procedure, prepare immobilization media for the ablation by dissolving $1.5 \%$ methylcellulose in $0.02 \%$ tricaine/E3. Store aliquots of methylcellulose for immediate use at room temperature, or at $4^{\circ} \mathrm{C}$ for long-term storage.

1. Anesthetize the $72 \mathrm{hpf}$ zebrafish by adding $5 \mathrm{mLs}$ of $0.2 \%$ Tricaine $\mathrm{pH} 7.0$ to the petri dish containing the zebrafish larvae in $30 \mathrm{mLs}$ of $\mathrm{E} 3$. The fish are anesthetized when they no longer exhibit the touch response. It is vital that the fish are completely anesthetized or they will twitch upon attempting to perform the laser ablation.

2. Examine the injected animals under the appropriate fluorescent setting and select animals in which you can easily visualize the proximal tubules.

3. Transfer selected embryos to a separate dish containing tricaine. Animals can be anesthetized for up to 30 minutes before performing cell ablation. If you score $>15$ animals, return these to a separate dish containing fresh modified E3 while awaiting ablation as each cell ablation takes several minutes.

4. To perform the ablation, transfer one anesthetized zebrafish to a small petri dish containing $1.5 \%$ methylcellulose/0.02\% tricaine for 2 minutes to wash the embryo in immobilization media.

5. Next, transfer the embryo to a glass depression slide containing a drop of $1.5 \%$ methylcellulose $/ 0.02 \%$ tricaine. Gently position the animal with a fine probe such that its ventral side faces the slide and dorsal side is facing up.

6. Place the slide on the compund microscope stage, and focus on the dorsal side of the animal. Perform ablation of the nephron cells under focus by depressing the foot pedal. You will see dispersal of the fluorescence as the renal epithelial cells are ablated (Figure 1A).

7. Gently transfer the ablated animal to a well in a 12 well-dish for subsequent culturing. Rinse the E3 2-3 times to wash away the methylcellulose.

8. After injection, raise the animal to the desired timepoint and perform desired analysis as per established histological and molecular techniques for young zebrafish embryos (Figure 2).

\section{Representative results:}

The data that are shown in Figure 1 indicate the ablation timecourse. After intramuscular injection with dextran-conjugates, the proximal tubule is preferentially labeled. Injection of dextran-FITC was used to label the kidney for laser ablation, and ablated animals were reinjected with dextranrhodamine at one additional timepoint following ablation to reimage the proximal tubule population. Expression analysis techniques like in situ hybridization can be used to analyze changes in fixed samples at single timepoints following cell ablation, as shown in Figure 2. 
A
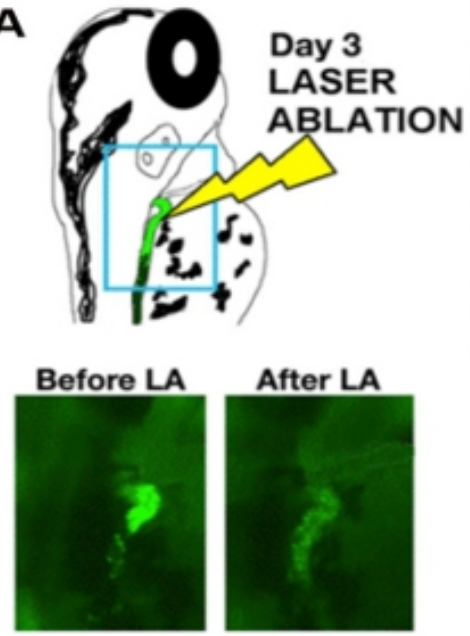

After LA

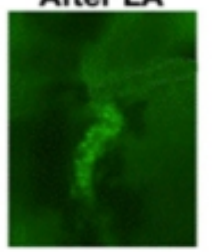

C

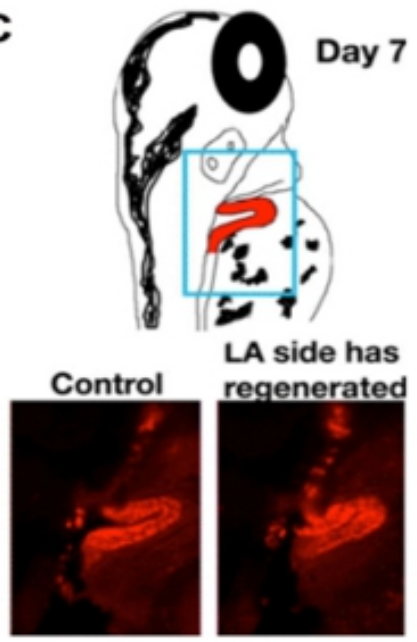

B

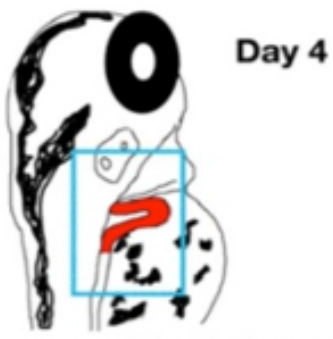

LA side has not

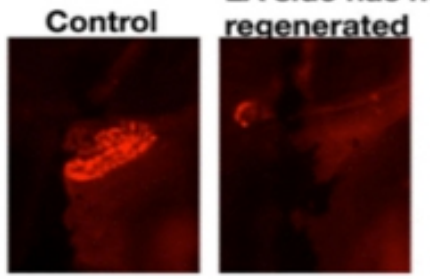

Figure 1: Laser ablation procedure is followed by rapid regeneration of the tubule epithelium. (A-C) Timecourse of cellular changes during and subsequent to laser ablation of zebrafish larva proximal tubules at time of ablation (day 3, panel A), or one or three days post ablation (day 4, panel B; day 7, panel C). (Top) schematics show position of the nephron, with dextran-FITC (green) and dextran-rhodamine (red), and (bottom) live images of control and laser-ablated (LA) nephrons. 
A

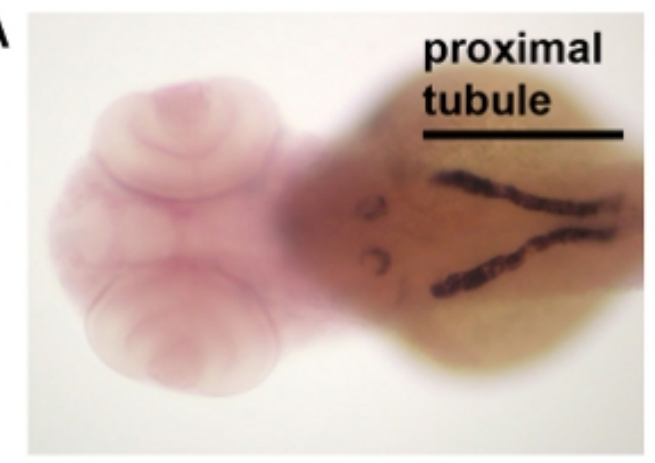

C

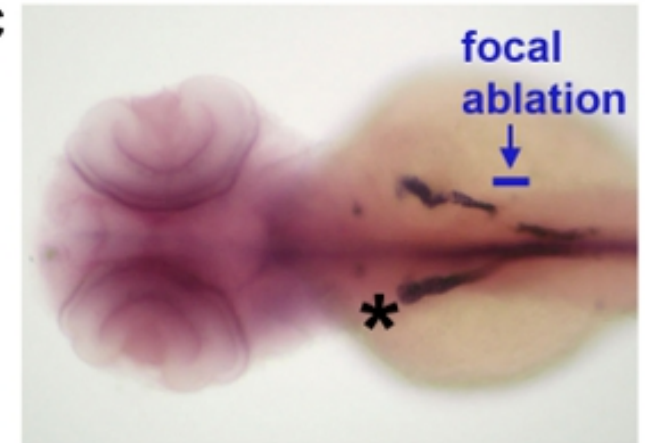

B

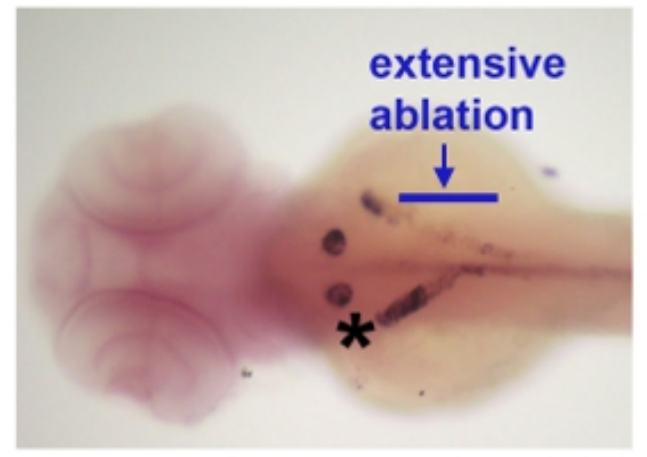

Figure 2: Analysis of gene expression following laser ablation. Following laser ablation at day 3, embryos were fixed and processed by whole mount in situ hybridization to detect transcripts for s/c20a1a, which mark the proximal convoluted tubule segment of the nephron. (A) A control embryo that was not subjected to laser ablation, (B) an embryo in which a large stretch of tubule epithelium was ablated, and (C) an embryo in which a short interval of tubule epithelium was ablated. Black line indicates the extent of the proximal tubule in $A$ to provide reference, blue lines indicate the extent of laser ablation in panels B-C, and * indicates the control (non-ablated) contralateral nephron in panels B-C.

\section{Discussion}

The zebrafish is a widely used model organism throughout many aspects of science, the applications of which are growing ${ }^{6}$. The employment of the zebrafish model system in the study of kidney diseases like AKI has led to important observations and will continue to be a good model to study kidney injury ${ }^{7,8}$. With a genome that has been sequenced and many molecular protocols in place, it has become increasingly easy to perform sophisticated zebrafish research that utilizes transgenic models, gene knockdown and misexpression studies. The zebrafish has a short lifecycle with large generation sizes and well-defined developmental stages. Furthermore, the zebrafish embryonic and larval stages are largely transparent, making imaging studies possible without dissection of the organism. Throughout embryonic and larval stages, the zebrafish has a pronephric kidney comprised of two nephrons, which remain visible during these developmental timepoints. Zebrafish pronephric nephrons are analogous in structure and function to their mammalian counterparts, making it a good model for acute renal failure studies ${ }^{4,5}$.

The kidney is crucial to the survival of humans and other vertebrates, serving as the organ that cleanses the body of liquid metabolic wastes. This cleansing function rests on the ability of the kidney nephrons to filter the blood, and then modify the filtrate to collect nitrogenous wastes for secretion and simultaneously maintain fluid and electrolyte balance. Nephrons are comprised of a blood filter, epithelial tubule, and drain into a duct. The structural and functional integrity of all three parts is integral to nephron function. Various insults to the kidney, including exposure to nephrotoxins, ischemia, or obstruction of the urinary outflow tracts can result in $\mathrm{AKI}^{1}$. The pathology of AKI is often associated with acute tubular necrosis 1,2 . Clinical studies have shown that ensuing renal failure has a mortality rate that can range anywhere from $7-80 \%$ depending on the cause and context of the renal failure. However, rapid diagnosis and reversal of the underlying cause of AKI is increasingly associated with recovery via regeneration of the necrotic nephron tubules. Recent fate mapping studies have shown that the major cell population that repopulates damaged nephron tubules are epithelial cells that originate from adjacent, uninjured areas ${ }^{12}$. These findings have not eliminated the possibility that renal stem/progenitor cells may participate in the process, and independent reports have suggested that bone marrow derived cells can contribute to renal regeneration ${ }^{13}$. It is clear that continued investigations are needed to better resolve cellular sources of renal epithelial regeneration.

Both nephron regeneration and development share the phenomenon of switches in cellular state between mesenchymal and epithelial phenotypes. During nephron development, mesenchymal progenitor cells differentiate into a stationary phenotype, attaching to a basement membrane to form the tubular epithelium ${ }^{3}$. The speculated migratory phenomenon of epithelial cells following acute renal failure necessitates a dedifferentiation into a mesenchymal phenotype. Interestingly, recent studies suggest that mesenchymal cells in damaged kidneys exhibit an expression profile similar to a mesenchymal cell during development ${ }^{14}$. Various reports have detected changes in cell adhesion molecules, matrix proteins, cytokines and chemokines. Following the proposed mesenchymal to epithelial transition, cells reassemble on a basement membrane and reinitiate tubular epithelial activities. Determining the genes important in this process continues to be a topic of research for our lab and others. Much of the work to elucidate the key factors in this process remains to be done, but significant progress in the field has been heralded by the study of the effects of gentamicin. 
$\mathrm{AKI}$ and renal failure caused by gentamicin is relevant considering the prevalence of nephrotoxic compounds used in medicine ${ }^{15}$. Used as a treatment for gram-negative bacterial infections, the administration of aminoglycosides leads to acute injury in $10-25 \%$ of cases. The drug causes a plethora of negative cellular effects, together resulting in apoptosis or necrosis in many tubular cells. Studies have found the drug to bind preferentially to a complex formed by megalin and cubulin that is involved in endocytosis. These molecules are found in abundance in epithelial cells of the proximal tubule, though they are not limited to expression in these cells. Through cellular signaling resulting in induction of oxidative stress, release of vasoconstrictors, depletion of cellular ATP, hypoxia, inhibition of phospholipases, gentamicin and similar antibiotics cause apoptosis and necrosis in epithelial cells. Additionally, antibiotics trigger mesangial contraction in the nephron blood filter (glomerulus), resulting in a drop in glomerular filtration rate and negatively altering the ability of the kidney to filter the bloodstream. The production of reactive oxygen species, immunostimulatory molecules, and activation of phospholipases have diffuse effects in the nephron, creating a catastrophic pathology that leads to acute renal failure.

While gentamicin and similar antibiotic studies have been and will continue to be important tools for kidney regeneration research, they lack a precision that can be useful in addressing certain questions. Our system of using the zebrafish in conjunction with a laser ablation method described in this protocol answers the need of such a precise research tool. Laser ablation allows researchers to induce cell destruction in focal areas of the renal tubule, ranging from a small (2-3) to large (>50-100) populations, with unrivaled precision. In this protocol, we utilize a previously developed method of exposure to dextran-conjugates to preferentially label proximal tubule epithelial populations. Alternatively, transgenic zebrafish lines that express green fluorescent protein in one or more regions of the tubule could be used. For example, cadherin 17:eGFP transgenic zebrafish exhibit strong fluorescence in distal tubule regions (though weak in proximal populations), and could be valuable for studies of regeneration in other tubule segments ${ }^{16}$. The visibility of the nephron will enable time-lapse video recording of cellular changes over time. When coupled with gene expression studies such as whole mount in situ hybridization or immunohistochemistry, researchers can detect molecular alterations in the nephron over time. Taken together, such strategies can begin to formulate a more dynamic understanding of the events that transpire when renal epithelial cells are destroyed.

The major caveat of our laser ablation model is that it may recapitulate partial aspects of the physiological conditions that transpire in human AKI. The cell death induced through instantaneous physical damage is different from the cascade of events that leads to necrosis in cells, and as such the humoral factors in these different microenvironments may not be identical. Additionally, there exists evidence of apoptosis induced during kidney injury, and it is not known whether such consequences transpire following insult with a laser. However, just as cell culture is a tool that better answers some questions that necessitate a highly controlled environment, laser ablation will serve as a highly controlled in vivo model of AKI. As such, the insights garnered from renal epithelial ablation studies in zebrafish will likely promote discovery of fundamental insights that can be applied to investigations of other renal injuries and potentially used to create better diagnostics in humans.

\section{Disclosures}

No conflicts of interest declared.

\section{Acknowledgements}

We thank members of the Wingert lab for useful discussions of AKI biology and zebrafish techniques, and C. Diep for sharing his methylcellulose recipe. The authors also wish to express our gratitude to the staff members of the Notre Dame Center for Zebrafish Research for providing excellent ongoing husbandry care for our zebrafish colony. Funding from the NIH-NIDDK grant award DK083512, and generous laboratory startup funding from the University of Notre Dame, supported this work.

\section{References}

1. Thadhani, R., Pascual, M., \& Bonventre, J.V. Acute renal failure. New Eng. J. Med. 334 (22), 1448-60 (1996).

2. Bonventre, J.V. Dedifferentiation and proliferation of surviving epithelial cells in acute renal failure. J Am. Soc. Nephrol. 14, S55-61 (2003).

3. Dressler, G.R. The cellular basis of kidney development. Annu. Rev. Cell Dev. Biol. 22, 509-29 (2006).

4. Wingert, R.A., Selleck, R., Yu, J., Song, H.D., Chen, Z., Song, A., Zhou, Y., Thisse, B., Thisse, C., McMahon, A.P., \& Davidson, A.J. The cdx genes and retinoic acid control the positioning and segmentation of the zebrafish pronephros. PLoS Genet. 3 (10), 1922-38 (2007).

5. Wingert, R.A. \& Davidson, A.J. The zebrafish pronephros: a model to study nephron segmentation. Kidney Int. 73 (10), 1120-7 (2008)

6. Lieschke, G.J. \& Currie, P.D. Animal models of human disease: zebrafish swim into view. Nat. Rev. Genet. 8 (5), 353-67 (2007).

7. Hentschel, D.M., Park, K.M., Cilenti, L., Zervos, A.S., Drummond, I.A., \& Bonventre, J.V. Acute renal failure in zebrafish: a novel system to study a complex disease. Am. J. Physiol. Renal Physiol. 288, F923-29 (2005).

8. Cianciolo Cosentino, C., Roman, B.L., Drummond, I.A., \& Hukriede N.A. Intravenous Microinjections of Zebrafish Larvae to Study Acute Kidney Injury. http://www.jove.com/index/Details.stp?ID=2079 doi: 10.3791/2079. J.Vis. Exp. 42 (2010).

9. Drummond, I.A., Majumdar, A., Hentschel, H., Elger, M., Solnica-Krezel, L., Schier, A.F., Neuhauss, S.C.F., Stemple, D.L., Zwartkruis, F., Rangini, Z., Driever, W., \& Fishman, M.C. Early development of the zebrafish pronephros and analysis of mutations affecting pronephric function. Development. 125, 4655-67 (1998).

10. Westerfield, M. The Zebrafish Book. 3rd ed (University of Oregon Press, Eugene, 2000).

11. Kemp, H.A., Carmany-Rampey, A., \& Moens, C. Generating chimeric zebrafish embryos by transplantation. http://www.jove.com/index/ Details.stp?ID=1394 doi: 10.3791/1394. J. Vis. Exp. 29 (2009).

12. Humphreys, B.D., Valerius, M.T., Kobayashi, A., Mugford, J.W., Soeng, S., Duffield, J., McMahon, A.P., \& Bonventre, J.V. Intrinsic epithelial cells repair the kidney after injury. Cell Stem Cell. 2 (3), 284-91 (2008).

13. Bussolati, B., Hauser, P.V., Carvalhosa, R., \& Camussi, G. Contribution of stem cells to kidney repair. Curr. Stem Cell Res. Therapy 4, 2-8 (2009).

14. Devarajan, P. Update on mechanisms of ischemic acute kidney injury. J. Am. Soc. Nephrol. 17, 1503-20 (2006). 
15. Lopez-Novoa, J.M., Quiros, Y., Vicente, L., Morales, A.I., \& Lopez-Hernandez, F.J. New insights into the mechanism of aminoglycoside nephrotoxicity: an integrative point of view. Kid. Int. doi: 10.1038/ki.2010.337 (2010).

16. Diep, C.Q., Ma, D., Holm, T., Naylor, R., Arora, N., Wingert, R., Bollig, F., Djordjevic, G., Lichman, B., Zhu, H., Ikenaga, T., Ono, F., Englert, C., Cowan, C., Hukreide, N.A., Handin, R.I., \& Davidson, A.J. Identification of adult nephron progenitors capable of kidney regeneration in zebrafish. Nature. 470 (7332), 95-100 (2011). 